\title{
Changer nos habitudes de prédation : l'exemple de la loutre et du pisciculteur
}

Elisabeth Rémy et Patricia Pellegrini

\author{
(2) OpenEdition \\ Journals \\ Édition électronique \\ URL : http://journals.openedition.org/ere/4187 \\ DOI : $10.4000 /$ ere.4187 \\ ISSN : 2561-2271 \\ Éditeur \\ Centr'ERE \\ Référence électronique \\ Elisabeth Rémy et Patricia Pellegrini, «Changer nos habitudes de prédation : l'exemple de la loutre et \\ du pisciculteur », Éducation relative à l'environnement [En ligne], Volume 5 | 2005, mis en ligne le 20 \\ novembre 2005, consulté le 21 février 2020. URL : http://journals.openedition.org/ere/4187 ; DOI \\ 10.4000/ere.4187
}




\title{
Changer nos habitudes de prédation : l'exemple de la loutre et du pisciculteur
}

\author{
Elisabeth Rémy et Patricia Pellegrini
}

Nous tenons à remercier Sylvie Lupton et Christina D’Alessandro pour leurs précieux conseils.

\section{Introduction}

1 Pour qu'une espèce puisse vivre et se déplacer librement, tel est le paradoxe, il faut multiplier les dispositifs et les aménagements techniques qui lui permettront de vivre à «l'état naturel» (Micoud, 1993). Pour mettre la nature à l'abri de l'être humain, ou inversement pour mettre les humains à l'abri d'un animal prédateur, il faut inclure davantage les humains et les animaux dans des dispositifs complexes. Notre étude de cas porte sur l'aménagement d'une pisciculture subissant les prélèvements importants de loutres avides de poissons. Au lieu d'éliminer l'animal, le pisciculteur, aidé de naturalistes, va tenter de trouver un compromis pour concilier son activité économique et la préservation de la faune sauvage. Cette expérience est d'abord l'occasion de nouvelles collaborations entre des acteurs agissant habituellement depuis des univers différents. L'aménagement de la pisciculture offre également l'opportunité de découvrir d'autres connaissances sur la loutre dans une dynamique où se croisent savoirs experts et savoirs " profanes ».

2 Comprendre toutes les astuces qui sont devenues nécessaires pour une cohabitation réussie entre être humain et loutre suppose de suivre, au plus près, les expérimentateurs et les difficultés qu'ils traversent au cours de leurs essais successifs (Stengers, 2000). Par quels moyens faire coexister sur un même espace l'être humain et l'animal prédateur? Comment va se comporter la loutre dans un environnement inhabituel? Ses réactions vont-elles à l'encontre des connaissances admises par les naturalistes? 
3 Le dispositif que nous allons étudier met à l'épreuve les habitudes des animaux et celles des humains pour tenter de les transformer. «Le pari de Thompson (un naturaliste), on s'en souviendra, nous conviait à miser sur la possibilité de transformer des habitudes : si nous changeons les nôtres, nous donnerons une chance aux animaux de transformer les leurs» (Despret, 2002). Si d'ordinaire le naturaliste ne voit pas la loutre - qui est nocturne en France et très difficile à observer dans la nature - la pisciculture offre une expérience grandeur nature qui oblige à questionner autrement l'animal. Dans cette trajectoire où des liens inattendus se négocient comment et à quel prix parvenir à cohabiter?

4 Nous avons pu retracer cette expérience par des entretiens auprès de l'ensemble des acteurs concernés; la visite de la pisciculture a également permis d'approfondir certaines questions. Notre objectif est donc de retracer l'ensemble de l'expérimentation qui fut nécessaire pour amener une possibilité de paix avec l'animal sauvage, à travers une série d'interactions qui n'est pas sans rappeler les programmes/anti-programmes de Latour (1993) ; en effet, à chaque fois que quelque chose lui sera opposé, la loutre trouvera une parade pour pénétrer dans la pisciculture jusqu'à l'obtention de l'arrangement final. Des formes d'agencements inédits se mettent en place au cours d'un processus où les acteurs acceptent d'apprendre les uns des autres - et d'apprendre de la loutre.

\section{La loutre : un animal très discret}

5 Comme le dit ce naturaliste, c'est joueur, c'est adorable et on a envie de la caresser. La loutre est un animal attirant dont la réputation du comportement ludique est entretenue par les articles des revues naturalistes qui lui sont consacrés. Pourtant, cette attitude qui lui est couramment attribuée ne manque pas d'interroger cet autre naturaliste : on dit qu'elle est joueuse, mais personne ne s'en rend compte parce qu'on ne la voit jamais. Du coup, c'est l'image qu'on en a. C'est même, selon les spécialistes, la traque de l'animal - chassé pour sa fourrure et piégé pour sa consommation de poissons - qui aurait induit un changement comportemental chez la loutre qui serait ainsi passée d'une activité diurne à une vie nocturne ${ }^{1}$.

6 Lors de moments rares et fugaces, le spécialiste peut apercevoir les loutres méfiantes à l'aube ou au crépuscule tandis que d'autres ne la voient jamais : on procède par pistage, c'est notre truc, la recherche des traces de pas et des indices attestant la présence de l'animal ; ce qui est plus difficile pour la loutre c'est qu'elle ne laisse que de petites épreintes ${ }^{2}$, et que certains passent des années sans la voir.

7 Les connaisseurs de loutres ne seraient-ils alors que des connaisseurs d'épreintes et d'empreintes? Une jeune naturaliste, engagée dans la réalisation de cartes de répartition faunistique ${ }^{3}$ nous expliquait que la quête des indices de loutre sans jamais la voir, contribuait précisément à l'intérêt qu'elle lui portait. N'est-ce pas quand même un peu frustrant de ne jamais voir l'animal ?

8 Non, non, c'est ça qui me motive. Je vois qu'elle est passée là et ça me fait quelque chose. Et quand l'empreinte est fraîche, c'est encore plus ... Je me dis qu'elle est passée il y a quelques heures. Signalons enfin que d'autres naturalistes ne sont pas de cet avis et adoptent des attitudes différentes en choisissant de s'intéresser à des espèces de loutres exotiques, précisément parce qu'elles vivent le jour et sont donc visibles. 
9 Apparaissent ainsi des relations discrètes entre l'être humain et l'animal : l'être humain ne voit pas l'animal, mais l'observe à distance et indirectement. En conséquence, ce n'est pas la confrontation avec l'animal observé dans la nature qui permet de connaître la loutre ; celle-ci est connue par l'image véhiculée par les revues naturalistes à travers des photographies ${ }^{4}$ ou des croquis, d'une part et de l'autre, par la quête des indices qu'elle laisse en chemin pour les naturalistes les plus motivés. La principale activité des naturalistes est, en effet, de réaliser des cartes de répartition sur la loutre par la recherche et la collecte de ses traces puisque celle-ci ne peut s'observer de visu.

\title{
Prendre conscience de la présence du prédateur
}

10 Dès lors, comment le pisciculteur peut-il se rendre compte de la présence de la loutre, cet animal que les naturalistes ne voient même pas au cours de leurs sorties de terrain? N'étant pas originaire de la région, celui-ci exerce son activité depuis cinq ans. Après ces études de pisciculture, il s'installe en Limousin avec une idée très précise de ce qu'il veut faire :

\begin{abstract}
Moi, la pisciculture achat/revente, ça ne m'intéresse pas, j'appelle ça de la poissonnerie. Ce qui m'intéressait, c'est d'avoir vraiment de quoi faire la reproduction, c'est le plus sympa du métier, c'est plus de boulot, mais c'est plus gratifiant quand les loutres ne s'en mêlent pas. C'est plus gratifiant parce que c'est votre poisson. Quand vous avez un client qui est content, c'est quand même vous qui l'avez fait de A à $\mathrm{Z}$, ce n'est pas un poisson que vous avez pris chez un collègue, que vous revendez.
\end{abstract}

Située à 707 mètres d'altitude sur le Plateau de Millevaches en bordure de la Petite Vézère, la pisciculture connaît des hivers rigoureux avec des températures atteignant souvent moins $10^{\circ} \mathrm{C}$. Elle se compose de cinq bassins en paroi bétonnée et de trois bassins de terrain, d'un bassin pour reproducteurs et d'un étang de pêche. Ignorant la présence de la loutre, le pisciculteur attribue d'abord les premiers signes du prédateur à une simple erreur de calcul :

C'était du poisson qui commençait à manquer à droite, à gauche. Vous savez ce que vous sortez comme alevins, comme œufs. Avant de remettre en bassins, vous pesez, vous faites un poids moyen et au bout de l'année, sur vos inventaires et les variations de stocks, etc., il y avait de plus en plus d'écart. La première année, déjà la loutre je ne m'en doutais pas. Donc quand on ne s'en doute pas, on se dit, je ne sais pas, j'ai mal compté au départ.

12 Comme l'ancien propriétaire finit par l'alerter sur le problème des loutres, le responsable des pertes est donc identifié. Le pisciculteur veut trouver une solution sans détruire l'animal parce qu'il est soucieux de la qualité de son environnement. Il entreprend alors de contacter divers organismes, la DDAF (Direction Départementale de l'Agriculture et de la Forêt), le CSP (Conseil Supérieur de la Pêche) puis le Centre Nature. Mais le pisciculteur a du mal à se faire entendre et certains naturalistes ne le croient pas :

Il [un naturaliste spécialiste des loutres] m'a répondu premièrement que ce n'était pas possible que les loutres prélèvent ou blessent tout ce cheptel, c'est une quantité trop importante parce qu'il est très théorie; deuxièmement qu'il connaissait bien la région et qu'il n'y avait pas tant de loutres que ça; troisièmement que les gens en Corrèze faisaient n'importe quoi, mettaient n'importe quoi, des truites et tout dans les étangs alors qu'elles crevaient. Alors, quand il m'a dit ça, j'ai dit «écoutez monsieur, au revoir » et puis point. [...] 
Donc X n'a jamais été réellement intéressé par le problème et en tout cas, il fallait se débrouiller, la loutre était là avant et il faut arrêter de dire tout un tas de choses sur la loutre, il n'y en a pas tant que ça, elle ne fait pas tant de dégâts, patati, patata.

Pour le pisciculteur, adopter une telle démarche, c'est aussi courir le risque de se voir rejeter par son milieu professionnel : ils se sont foutus de moi, ils m'ont dit, mais qu'est-ce que tu t'embêtes; maintenant tu vas être surveillé, tu les flingues [...], de toute façon tu n'auras jamais rien. Si je n'avais rien obtenu, ils seraient en train de rigoler, comme il faut. D'ordinaire, les autres pisciculteurs n'ont effectivement ni la même approche ni les mêmes scrupules et les animaux sont parfois tués lorsqu'ils s'aventurent sur de nouveaux territoires. Une loutre vient trop souvent sur une pisciculture, le pisciculteur va faire justice lui-même. Il ne va rien dire à personne, il sait pertinemment que c'est protégé. Cependant, une évolution semble perceptible puisque certains de ses collègues se renseignent maintenant sur ce qu'il est possible de faire en pareil cas ; comme on le verra plus loin, la pisciculture devient, en quelque sorte, la vitrine des solutions possibles.

Du point de vue écologique, les spécialistes considèrent que la mort de l'animal ne règle que temporairement le problème parce que si le secteur est vide, une autre loutre viendra occuper la place de l'animal précédent pour, à son tour, profiter du « restaurant à loutre ». L'expérience vise justement à commencer une démarche qui, au lieu de détruire les animaux au fur et à mesure de leur progression, vise à trouver des mesures techniques efficaces; l'objectif est d'éviter que les loutres portent préjudice aux productions des humains lesquels, en retour, respectent l'animal à condition de canaliser ses déplacements.

Cette fois, les démarches du pisciculteur ne restent pas vaines. Un médiateur Faune sauvage appartenant au monde associatif prend en charge l'opération pour trouver une solution aux multiples prélèvements effectués dans les bassins de salmonidés. Il rédige un rapport en septembre 1999 dans lequel il fait ce premier constat: «Le préjudice s'avère non négligeable, estimé à près de $500 \mathrm{~kg}$ de poissons reproducteurs sur l'année [...]. À titre de comparaison, une loutre en captivité consomme quotidiennement 850 à $1200 \mathrm{~g}$ de poissons » (Leblanc, 2001).

Nous ferons, plus loin, un point sur la façon dont cette expérience bouscule les connaissances admises chez les naturalistes, mais avant il est important de voir comment la loutre va réagir aux différents dispositifs d'effarouchement qui vont lui être opposés.

\section{Tester les systèmes d'effarouchement de la loutre}

Le médiateur faune sauvage et le pisciculteur se lancent alors dans des expérimentations qui amènent chacune leur lot de surprises :

Cela [l'éclairage avec déclencheur] a marché parce que moi, de ma cuisine, je vois bien la pisciculture et toute la nuit c'était Versailles. Je me disais «ça marche, elles montent, ça éclaire, elles redescendent [...]». Le lendemain, tous les restes de poissons étaient au pied des phares.

Après, on a renforcé la clôture, on a mis des répulsifs olfactifs sur les points de passage. Ce sont des répulsifs pour les chiens, les chats, ça marche assez bien, cela dégage une odeur assez forte, désagréable, d'œuf pourri. Effectivement, elles reniflent, mais au bout d'un moment elles venaient crotter un peu à côté.

On pensait que ça marchait parce qu'elles ne passaient plus à ces endroits-là [...] jusqu'à ce qu'on trouve les nouveaux passages [...]. 
On avait essayé de mettre des pièges photographiques, il n'y a eu que moi et mon chat en photo ! [...] Il fallait vraiment qu'elle passe pile-poil sur la palette. C'est vrai que pour nous $-20^{\circ}$, c'est une routine. Les palettes se gelaient, si on mettait des cellules photo à déclenchement automatique, avec l'humidité il fallait les mettre dans des boîtes étanches. Bref, c'était très compliqué, ce qui fait que ça ne marchait pas.

Les expérimentateurs installent alors des clôtures électriques. Mais, la loutre parvient toujours à passer sans toucher le fil situé au ras du sol. Au bout de combien de temps, l'animal trouve-t-il une parade pour contourner les obstacles dressés successivement sur son chemin?

Pour trouver une autre solution? C'était quelques jours. Elles devaient se faire cogner un petit peu au début parce que la truffe doit être humide, mais, au bout d'un moment, soit elles passaient en force et, en une seconde, elles traversaient la clôture ; soit elles creusaient dessous et elles passaient ; ou elles trouvaient un autre point d'attaque. On ne pouvait pas électrifier tout le long de la pisciculture. On essayait de boucher avec des pierres régulièrement, mais elles trouvaient toujours un point de passage. Après, on s'est aperçu, une fois que c'était bien clôturé, qu'il est possible qu'elles montent aussi par les arbres. Un naturaliste est venu nous voir et il a pris au sérieux notre problème. Il a dit «il n'y a pas de problème, elles grimpent ici ».

Si l'animal n'a évidemment pas l'agilité d'un écureuil, il est capable de grimper aux arbres dont les écorces très profondes lui offrent des prises.

Pendant toute cette phase d'essais s'échelonnant sur 18 mois, les prélèvements continuent. Comme l'a déjà souligné Leblanc (2001) bien que les techniques mises en œuvre (grillage, clôture électrique, par exemple) soient similaires à celles utilisées dans les zoos, la problématique défendue ici est inversée: d'une part, il ne s'agit pas d'empêcher la loutre de sortir, mais de l'empêcher d'entrer, d'autre part c'est l'être humain et non l'animal qui se retrouve encerclé de grillages.

21 Le journal de bord du médiateur faune sauvage dresse un tableau impressionnant des prédations effectuées par la loutre, montrant en retour sa capacité d'adaptation et sa résistance aux diverses techniques d'effarouchement employées :

Constatation de prélèvements de septembre 1999 à avril 2000

Les pertes chiffrées s'élèvent à $400 \mathrm{~kg}$ de poissons reproducteurs, et à $400 \mathrm{~kg}$ de poissons commercialisables. En 1999, en douze mois d'exploitation, 196 à 280 reproducteurs ont été prélevés. La perte financière est estimée de $80000 \mathrm{FF}$ à 90000 FF. Étant donné la perte de reproducteurs, le pisciculteur a dû pallier le manque par l'achat d'alevins et de truitelles. (Leblanc, 2001)

Alors que les prédateurs en situation ordinaire mangent entièrement le poisson qu'ils capturent, ici la loutre n'en mange que les meilleures parties, occasionnant ainsi des pertes très importantes. Devant l'abondance en nourriture qu'offre la pisciculture, seules les têtes des poissons sont consommées. Ce comportement est mal vécu non seulement en raison des pertes réelles qu'il provoque, mais aussi du sentiment d'avoir affaire à des animaux cruels : ça va être d'autant plus impopulaire, un peu ce qui se disait avant "ils tuent pour le plaisir». On va leur prêter des sentiments un petit peu anthropomorphiques. Dans la pisciculture, les dégâts sont d'autant plus conséquents qu'aux prises directes effectuées par l'animal s'ajoute l'affolement des poissons qui se 
jettent contre les bords des bassins, se blessent et sont donc impropres à la consommation.

D'ultimes aménagements (Leblanc, 2001) sont donc indispensables pour se prémunir de la prédation de la loutre :

- renforcement de la clôture électrique (3 fils) par un grillage torsadé ;

- utilisation d'un répulsif anti-carnassiers (schwegler);

- installation de bavettes de caoutchouc aux points d'entrée et de sortie des eaux ;

- consolidation du grillage : soit enterrer le grillage quand cela est possible (environ $30 \mathrm{~cm}$ ), soit l'insérer dans un socle en béton d'une hauteur de 30 à $40 \mathrm{~cm}$.

Comme on l'a vu précédemment, les premiers essais d'effarouchement ont un faible impact sur la loutre qui apparait moins craintive qu'on pourrait le supposer. Au cours de cette expérience, les dispositifs techniques sont aussi soumis à rude épreuve et la dureté de l'hiver a raison de la clôture électrique qui commençait à freiner les prélèvements. On pose aussi des bavettes en caoutchouc pour barrer l'entrée par le canal, mais, cette fois, c'est le courant qui finit par les arracher; tous les éléments du dispositif font désormais partie de l'expérimentation. La loutre creuse sous le grillage puis parvient à sauter par-dessus les fils électriques, les dispositifs se compliquent en conséquence, mais la loutre passe toujours. C'est maintenant un véritable arsenal qui tente de mettre la pisciculture à l'abri des loutres; il faut concilier les astuces des loutres, leur gourmandise, les intérêts du pisciculteur et son bien-être, au risque d'enfermer le pisciculteur dans un véritable blockhaus encombré de grillages qui gênent l'entretien quotidien de ses bassins piscicoles.

Ces expérimentations mettent aussi en évidence les implicites que les naturalistes mobilisent dans une situation comme celle-ci ; partant de l'idée que la loutre n'apprécie pas l'inattendu, on suppose qu'elle sera effrayée par quelque chose qui surgit brusquement. Comme elle vit désormais la nuit, on lui impose des jets brusques de lumière, mais rien n'y fait, la loutre résiste venant même manger sous la cellule du projecteur.

26 Pendant près de deux ans, le pisciculteur a donc accepté de se livrer à ces diverses expérimentations tout en continuant à subir cette prédation puisque les divers dispositifs testés sur place ont montré leurs limites. À chaque fois, il a fallu innover pour contrecarrer les intrusions persistantes de l'animal. L'arrangement final s'obtient en négociant avec la qualité du sol, les rigueurs de l'hiver, le degré de résistance des matériaux, l'humidité, l'intensité du courant et surtout la ruse de la loutre.

Pour que le souhait du pisciculteur se réalise, il a fallu expérimenter toute une série de mesures techniques; le conflit de la loutre et du pisciculteur est à la fois déplacé puis pacifié par l'ajout d'astuces techniques (grillage, béton, par exemple) qui engagent autrement et ensemble humains et non-humains. C'est d'abord l'association du pisciculteur et du médiateur faune sauvage, puis l'intéressement de la DIREN (Direction Régionale de l'Environnement), puis l'obtention de financements, puis des grillages et autres répulsifs qui redonnent aux expérimentateurs leur vie normale - après les avoir transformés en veilleurs de nuit; et c'est cet ensemble de liens éprouvés les uns à la suite des autres qui finit par aboutir à l'accord: le pisciculteur travaille en paix, la loutre passe sans trépasser. 


\title{
Autre dispositif, autre comportement
}

\section{plutôt à l'encontre des rares observations des naturalistes qui d'ordinaire ne voient pas} la loutre :

\begin{abstract}
Le fait que plusieurs loutres pêchent sur une même pisciculture a été constaté à plusieurs reprises. Les animaux sont alors peu discrets. Leur nombre peut être de 3 à 5 individus présents simultanément sur le site pendant deux à trois heures. Il pourrait s'agir d'une famille constituée de la femelle et de ses deux jeunes de l'année, voire d'individus un peu plus âgés ayant 2 ans ou plus.

La présence périodique du pisciculteur et de son chien au cours de la nuit ne semble pas gêner l'animal (ou les animaux). La loutre suspend alors son activité de chasse pour la reprendre une fois que le pisciculteur a achevé sa visite des bassins. (Leblanc, 2001)
\end{abstract}

Cette expérience met ainsi à l'épreuve les connaissances et les présupposés des naturalistes sur la loutre : on la dit solitaire, voilà que cinq loutres sont capables de se retrouver en même temps sur le même lieu; on la dit farouche, voilà qu'elle s'habitue rapidement aux techniques censées l'effrayer; on la dit de faible appétit, la voilà prise en flagrant délit de gloutonnerie; on la dit, opportuniste allant au plus facile, et pourtant elle n'hésite pas à affronter toutes sortes de difficultés - creuser, recevoir des décharges électriques, grimper, se faire flashée, etc. - pour accéder à la nourriture qu'elle ne trouvera, certes jamais, avec une telle abondance dans la nature. Si elle évite la rencontre avec le pisciculteur et son chien, elle n'hésite pas à revenir une fois la ronde effectuée. On peut supposer que l'on découvrirait autre chose sur le comportement de l'animal si on multipliait les observations nocturnes dans des contextes qui diffèrent de ceux généralement fréquentés par les naturalistes.

L'exemple de l'aménagement de cette pisciculture montre que les flashs lumineux ne contrarient pas la loutre désireuse de s'approvisionner dans les bassins d'élevage. Un débat du même type porte sur le fait de savoir si le flash du piège photographique utilisé dans les passages à loutre sous les autoroutes ${ }^{5}$ dérange ou non l'animal :

C'est un débat de spécialistes. À mon avis, tout se joue à la première fois. La première fois qu'il passe, il est flashé : soit il peut considérer que jamais plus il ne repassera par là, soit il peut se dire que finalement il ne s'est rien passé et qu'il peut réessayer. Encore une fois, c'est un raisonnement qui est très humain.

Une fois passée leur première surprise, la loutre semble donc s'habituer à ce que les humains ont eu la courtoisie de construire pour elle. Mais finalement qu'il s'agisse de vérifier par des prises photographiques qu'elle utilise les passages à faune qui lui sont destinés ou qu'il s'agisse de les dissuader d'entrer dans la pisciculture, cet animal fait bien peu de cas des jets de lumière qu'on lui inflige. Nocturne certes, il s'accommode très bien de la lumière et même d'être sous les feux des projecteurs, pourvu qu'il y ait des poissons à dévorer.

Au terme de cette expérience, les loutres deviennent moins méfiantes. Le pisciculteur a pu s'approcher des loutres et celles-ci s'habituent à la présence de l'être humain pacifique montrant même un certain degré d'anthropophilie :

On les voyait venir à trois à quatre heures du matin et elles repartaient juste avant le lever du soleil. Et sur les derniers temps, à sept heures et demie du soir, en été, on en a regardé pendant vingt minutes avec $\mathrm{X}$, deux sur les cordes de la passerelle, une qui jouait dans les courants sans se sauver ${ }^{6}$. 
Après toute une série d'ajustements, les dispositifs parviennent enfin à maintenir les loutres au-dehors et le pisciculteur est lui-même devenu un pisteur de loutre sachant décrypter ses indices de présence :

Je n'entends plus siffler la nuit et même aux abords de la pisciculture, l'herbe n'est pas couchée au matin. Au début, elles longeaient les nouveaux grillages, elles ont cherché. On voyait l'herbe couchée. C'est pour ça, entre autres, que toute cette partie-là, je ne l'ai pas tondue parce que je continue à regarder un peu ce qui se passe. Je pense qu'elles se sont réparties sur un territoire beaucoup plus vaste.

Se pose alors la question du transport des connaissances d'un contexte à l'autre ; les connaissances habituelles sur l'invisible loutre viennent essentiellement du pistage des indices qu'elle laisse en chemin (empreintes et épreintes). C'est précisément parce qu'elle n'évolue pas dans son élément habituel - en l'occurrence dans une pisciculture et qu'elle devient cette fois visible - que la loutre interroge les connaissances des naturalistes et les oblige à réviser ce qu'ils pensaient d'elle.

\section{Qui doit supporter le coût de cette cohabitation?}

Sans pouvoir entrer, dans le cadre de ce texte, dans un débat approfondi sur la place de la prédation dans un milieu anthropisé, cette étude de cas pose néanmoins la question de savoir qui doit supporter le coût de cette cohabitation. "Pour les agriculteurs en effet [...] l'introduction d'une espèce animale nouvelle se traduira souvent par une nuisance, qu'il est légitime - et quant au succès même de l'opération, recommandé d'indemniser " (Pelosse, 1992). Ce souci ${ }^{7}$ est partagé par des naturalistes qui souhaitent même anticiper le problème avant qu'il ne devienne trop important (Reuther, 1996). Ce coût semble au départ uniquement reporté sur le pisciculteur et ses demandes de subvention auprès des divers organismes concernés restent vaines au motif que les loutres étaient là avant lui :

C'est pour ça que j'en étais arrivé à téléphoner au Ministère de l'Environnement et ils m'ont carrément envoyé promener. Ils m'ont dit «il n'y a rien, mais attention, qu'on ne vous prenne pas à en piéger une !». Il fallait laisser faire, il fallait que je me taise, c'était protégé, mais aucune contrepartie. Je voulais bien rester sur mes idées de protection de l'environnement, mais j'attendais un appui de quelqu'un.

Le problème se pose, de la même façon, pour la Chambre d'Agriculture qui ne peut apporter aucune aide: ils n'ont pas de fonds pour ça. Il n'y a pas non plus d'assurance qui existe contre la prédation c'est-à-dire que tout ce qui s'en allait, c'était de la perte pure. Face à ce type de problème, l'Administration en charge de l'environnement, en région et au niveau central, craint de créer un précédent : la DIREN nous avait clairement dit "pas d'indemnisation». Pour eux, c'était une fuite en avant sinon tous les pisciculteurs allaient demander pour les cormorans, pour le héron, pour le martin-pêcheur. Selon la DIREN, cette réflexion sur les dégâts occasionnés par les loutres devrait être menée au niveau national. Les réticences à l'indemnisation, au-delà des problèmes de moyens qu'elle pose, s'expliquent aussi par le risque de se faire rembourser des pertes que l'on imputerait abusivement à la loutre.

L'association Limousin Nature Environnement propose alors de signer une convention avec l'exploitation piscicole pour qu'il y ait des engagements de part et d'autre: les premiers s'engagent à soutenir le dossier au niveau de la DIREN ; les seconds à valoriser cette expérience de site pilote visant par exemple à accueillir les classes en formation 
aquaculture ou les jeunes pisciculteurs désirant s'installer. Mais reste encore à trouver les financements et surtout à déterminer ceux qui devront prendre en charge le coût de cette activité économique réalisée avec le souci de préserver la nature et les espèces sauvages. À la suite de nombreuses démarches pour trouver les financements et convaincre les divers responsables concernés (diverses commissions des DIREN, Trésorerie Générale), le pisciculteur qui avait déjà subi des pertes financières importantes, obtient finalement une aide pour la réfection entière de la pisciculture. Les coûts des travaux s'élèvent à 200000 francs environ (30 500 euros). En janvier 2002, la convention a été signée entre la pisciculture et Limousin Nature Environnement pour effectuer les travaux de protection de la pisciculture avec les procédés testés sur place.

38 Ce lien entre activités économiques et préservation de la nature, tant prôné par le développement durable, trouve ici une réalisation concrète que le pisciculteur est prêt à faire connaître afin de montrer aux gens que l'Environnement n'est pas là que pour mettre des interdits. Notons enfin que ce principe de concilier une activité socio-économique avec la préservation d'une espèce protégée rejoint la philosophie d'action de la directive Habitats Faune Flore prônant justement la préservation des espaces et des espèces qu'ils abritent tout en tenant compte des contraintes économiques présentes sur le site. La pisciculture aurait donc pu entrer dans le cadre de l'application de cette directive européenne en justifiant l'inscription du site par la présence de la loutre inscrite dans son annexe II. Si cela ne s'est pas fait, c'est pour éviter de créer un précédent qui se traduirait inévitablement par des demandes de subventions que la DIREN ne peut pas prendre en charge : Oui, mais ils vont demander des sous. On va les avoir ponctuellement une fois. Si on a dix pisciculteurs qui nous demandent $250000 \mathrm{~F}$, on ne pourra pas. Il faut trouver après d'autres sources de financement. Une fois la solution apportée au problème, l'attention se déplace et elle peut, par retour d'expérience, servir de référence pour d'autres problèmes du même genre; le médiateur ou la DIREN savent qu'ils peuvent désormais apporter des conseils pour régler ce type de situations nonobstant l'obtention des fonds nécessaires.

\section{Conclusion}

L'aménagement de cette pisciculture montre les astuces qui sont rendues indispensables pour parvenir à vivre ensemble. La loutre ne peut pas cohabiter avec une pisciculture ordinaire ou plutôt le pisciculteur ne peut cohabiter durablement avec une loutre trop gourmande au risque de mettre en péril son activité. Au terme de l'expérimentation, la loutre "détournée » stabilise la DIREN, le pisciculteur, les associations de protection de la nature et des enjeux socio-économiques en évitant les conflits perpétuels entre producteurs et prédateurs qui se soldent souvent par le sacrifice de l'animal.

Tout le monde a changé et appris de et par ce dispositif : la loutre apprend à déjouer les obstacles dressés sur son chemin et le pisciculteur associé au médiateur faune sauvage recueille une connaissance experte et inédite sur la loutre. Ces derniers ont pu observer directement la loutre alors que les naturalistes n'ont souvent vu que des traces de son existence. De même, la loutre appréhende autrement le pisciculteur au point de se laisser observer à quelques mètres, montrant en cela qu'elle a appris à ne plus le craindre. 

loutre-gourmande oblige les humains à questionner autrement l'animal. Parce qu'ils n'ont pas d'observations de visu de la loutre, les naturalistes sont aussi plus prompts à accepter qu'elle puisse se comporter autrement.

ors, d'autres formes d'attachement avec l'animal sauvage sont repérables: les humains offrent à l'animal une chance de survie et la contention de la gloutonnerie de la loutre donne aux humains la possibilité de créer un monde différent. Chacun a modifié ses habitudes pour vivre en paix : le pisciculteur ne tue plus la loutre comme autrefois, la loutre ne vient plus manger les poissons d'élevage, animaux et humains se partagent le même lieu qui n'est plus tout à fait le même, car des moyens de dérivation comme les grillages obligent la loutre à faire des détours; de nouveaux espaces se créent et d'autres se modifient : c'est le résultat de la présence active de la loutre dans des espaces socialisés, mais aussi de l'influence de ces derniers dans le devenir de la loutre. La cohabitation d'intérêts divergents n'est possible qu'en posant des limites; cette délimitation créée des lieux inhabituels où l'on peut être, à la fois, ensemble (le long et autour de la rivière) et isolés (en dedans ou en dehors de la pisciculture) créant ainsi une nouvelle configuration spatiale.

Par la voix de ses divers porte-parole, la loutre est arrivée à se lier autrement avec les humains : elle ne va plus se nourrir dans les bassins d'élevage et, en échange, elle n'est plus tuée; le dispositif est parvenu à pacifier ce que cette rencontre risquait de compromettre en assurant la survie économique de l'un et la propre survie de l'autre. On peut donc dire que ces animaux partagent désormais des intérêts communs avec les humains parce que les uns et les autres sont plus satisfaits dans cette nouvelle configuration : le pisciculteur protège sa production piscicole, la loutre a la vie sauve ce qui contente aussi les humains impliqués dans cette histoire - et elle n'est plus sur ses gardes lorsqu'elle s'aventure sur cet espace puisqu'elle se laisse même observer par le pisciculteur qui est, lui-même, épaté par son caractère habile et rusé.

Si d'ordinaire le naturaliste ne voit pas la loutre, la pisciculture offre une expérience grandeur nature qui permet d'observer cet animal dans un contexte particulier, ce qui permet d'affiner les connaissances sur son comportement. La loutre change montrant ainsi un comportement assez flexible bien qu'elle conserve certaines exigences. Les naturalistes se sont d'abord intéressés à la loutre pour elle-même pour ensuite travailler à la cohabitation de la loutre avec d'autres. La pisciculture a été le support d'une épreuve qui permet de (ré)concilier humains et non-humains et ce à quoi nous sommes sensibles a changé (Despret, 2002).

Plus généralement, ce changement, observé sur un cas précis, semble préfigurer de nouvelles alliances dans notre rapport au vivant où il s'agit d'expliciter la liste des êtres avec qui l'on peut vivre ou ne pas vivre pour définir ensemble l'espace des simultanéités (Latour, 2005). 


\section{BIBLIOGRAPHIE}

Bouchardy, C., Rosoux, R. et Boulade, Y. (2001). La loutre d'Europe. Histoire d'une sauvegarde. Clermont-Ferrand : Catiche Productions-Libris.

Despret, V. (2002). Quand le loup habitera avec l'agneau. Paris : Les empêcheurs de penser en rond / Le Seuil.

Latour, B. (1993). Le fardeau moral d'un porte-clefs. Dans Latour, B. (dir.), La clef de Berlin et autres leçons d'un amateur de sciences (p. 47-55). Paris : La Découverte.

Latour, B. (2005). De la politique du temps à la politique de l'espace? Dans Rémy, E., November, V., D’Alessandro-Scarpari, C. et Charvolin, F. (dir.), Espaces, savoirs et incertitudes (p. 5-12). Paris : Ibis Press.

Leblanc, F. (2001). Prédations sur les installations piscicoles en Limousin : analyses des systèmes de protection. Rapport 2000/2001. Limousin : Limousin Nature Environnement.

Micoud, A. (1993). Vers un nouvel animal sauvage : le sauvage « naturalisé vivant »? Natures, Sciences, Sociétés, 1(3), 202-210.

Pellegrini, P. (1995). Zoos, parcs et réserves. Quel est le statut de ces animaux offerts au regard de l'homme? Dans Lizet, B. et Ravis-Giordani, G. (dir.), Des bêtes et des hommes (p. 227-242). Paris : CTHS.

Pelosse, V. (1992). Réintroductions d'espèces et agriculture : de l'anthropisation à l'artificialisation. Économie rurale, 208-209, 101-104.

Rémy, E. (1995). L'élaboration d'un savoir naturaliste. Approche anthropologique d'un atlas. Études rurales, 137, 55-72.

Rémy, E. (2005). Comment rendre visible l'invisible, deux façons de tracer la présence de la loutre? Communication présentée au colloque « Citizen sciences collective vigilance and relations between laymen and scientists in natural history », janvier 2005, Saint-Étienne.

Reuther, C. (1996). European otter habitat network. Dans Conseil de l'Europe (dir.), Seminar on the Conservation of the European Otter (Lutra lutra), Leeuwarden, Pays-Bas, 7-11 juin 1994 (p.188-193). Strasbourg : Publications du Conseil de l'Europe.

Rosoux, R. (1998). Étude des modalités d'occupation de l'espace et d'utilisation des ressources trophiques chez la loutre d'Europe (Lutra lutra) dans le marais poitevin. Thèse de doctorat d'État inédite, Université de Rennes 1.

Stengers, I. (2000). Discipline et interdiscipline : la philosophe de «l'écologie des pratiques ». Nature, Sciences, Sociétés, 8(3), 51-58.

\section{NOTES}

1. Sur la connaissance de la loutre, se reporter à la thèse de R. Rosoux (1998). Précisons que si l'espèce était considérée comme rare depuis les années cinquante, sa protection totale n'est intervenue qu'avec l'arrêté ministériel du 17 avril 1981. La loutre est désormais protégée par un ensemble de réglementations internationales au point que la convention de Berne l'ait même choisie pour emblème.

2. Nom donné aux crottes de loutres. 
3. Pour une première analyse anthropologique de la réalisation d'un atlas de répartition faunistique, se reporter à Rémy (1995).

4. Généralement, il s'agit soit de loutres exotiques soit de loutres vivant en captivité. Pour une réflexion sur le statut des animaux vivant dans les zoos, voir Pellegrini (1995).

5. Cette question a fait l'objet d'une présentation au colloque de Saint-Étienne sur les sciences citoyennes, organisé par F. Charvolin, voir Rémy 2005.

6. Bouchardy et coll. (2001) signalent qu'autrefois les loutres étaient apprivoisées pour la pêche.

7. Même s'il s'agit ici de l'expansion d'un animal déjà présent, le processus est similaire.

\section{RÉSUMÉS}

Notre étude de cas porte sur l'aménagement d'une pisciculture subissant les prélèvements de loutres avides de poissons. Cette expérience est l'occasion de nouveaux apprentissages entre des acteurs agissant habituellement depuis des univers différents. Comprendre toutes les astuces qui sont devenues nécessaires pour une cohabitation réussie entre être humain et loutre suppose de suivre, au plus près, les expérimentateurs et les tourments qu'ils traversent (Stengers, 2000). Pour qu'une espèce puisse se déplacer librement, tel est le paradoxe, il faut multiplier les aménagements techniques qui lui permettront de vivre à «l'état naturel » (Micoud, 1993). Nous montrerons alors que tous les protagonistes ont changé, car ils ont appris de et par ce dispositif. Chacun a modifié ses habitudes pour vivre en paix et créer ainsi l'espace d'une cohabitation.

Our case study deals with the management of a pisciculture suffering from fish takings from hungry otters. This experiment offers new learning opportunities to actors that usually carry out action from their different universes. The thorough understanding of the underlying factors that enable a successful cohabitation between human beings and otters requires a very close monitoring of the experiences and the troubles faced by the actors (Stengers, 2000). So that a species can move freely, such is the paradox, it is necessary to multiply technical installations that will enable the species to live in its "natural state" (Micoud, 1993). We will show that all protagonists changed because they learned from and through this device. Everyone modified their practices to live peacefully and thus create space for cohabitation.

\section{AUTEURS}

\section{ELISABETH RÉMY}

Travaillant sur les controverses dans le domaine de l'environnement et des risques, elle s'intéresse notamment à la confrontation des connaissances et des savoirs mobilisés en situation d'incertitude. Dans le domaine des sciences naturalistes, son intérêt porte plus précisément sur les procédures d'inventaire, la réalisation d'atlas faunistique et sur la notion de réseaux écologiques.

\section{PATRICIA PELLEGRINI}

Anthropologue de formation, ses recherches portent sur la construction des savoirs - tant scientifiques que vernaculaires - sur la « nature » dans les sociétés occidentales. Elle mène 
notamment une réflexion sur nos rapports aux animaux et les catégories, sauvages, domestiques, etc. auxquelles nous les renvoyons. 